Relations industrielles

Industrial Relations

\title{
Pfeffer, Jeffrey, Competitive Advantage Through People : Unleashing the Power of the Work Force
}

\section{François Villeneuve}

Volume 52, numéro 2, 1997

URI : https://id.erudit.org/iderudit/051179ar

DOI : https://doi.org/10.7202/051179ar

Aller au sommaire du numéro

Éditeur(s)

Département des relations industrielles de l'Université Laval

ISSN

0034-379X (imprimé)

1703-8138 (numérique)

Découvrir la revue

Citer ce compte rendu

Villeneuve, F. (1997). Compte rendu de [Pfeffer, Jeffrey, Competitive Advantage Through People : Unleashing the Power of the Work Force]. Relations industrielles / Industrial Relations, 52(2), 463-466.

https://doi.org/10.7202/051179ar

Tous droits réservés @ Département des relations industrielles de l'Université Laval, 1997
Ce document est protégé par la loi sur le droit d'auteur. L’utilisation des services d'Érudit (y compris la reproduction) est assujettie à sa politique d'utilisation que vous pouvez consulter en ligne.

https://apropos.erudit.org/fr/usagers/politique-dutilisation/ 
un sous, pour répondre aux récriminations de Sebastiano Brusco qui, soi-disant, prétend pouvoir nous mettre sur la piste des assassins d'Olaf Palme (p. 146). C'est là que l'ouvrage décroche sa plus mauvaise note. Mais il ne faudrait pas exagérer la portée de cette critique. On parle ici d'ajustement du tir. Le contenu en informations de l'ouvrage est impressionnant. On observe, en outre, rarement un tel niveau de cohérence à l'intérieur d'ouvrages mettant à contribution près d'une vingtaine d'auteurs, ce qui est tout à l'honneur des éditeurs. C'est un livre enrichissant à parcourir pour ceux qui s'interressent aux districts industriels italiens, et même pour les néophytes, compte tenu de ce niveau de cohérence.

BENOÎT MARTO PAPILLON

Université du Québec à Trois-Rivières

\section{Competitive Advantage Through People: Unleashing the Power of the Work Force}

by Jeffrey PFEFFER, Boston: Harvard Business School Press, 1994, 281 p., ISBN 0-87584-413-8.

Jeffrey Pfeffer soutient qu'il existe désormais une nouvelle source d'avantage concurrentiel à long terme, un facteur crucial de différenciation et difficile à imiter : la main-d'œuvre et la manière dont elle est gérée. Pourquoi je recommanderais la lecture de ce livre? Parce que dans mon rôle de gestionnaire, j'ai été secoué : mes héros, mes théories, mes habitudes et même mon langage sont remis en question par les arguments et le style provocateur de l'auteur. Comme consultant, je me suis délecté : dans un langage clair et accessible, l'auteur prescrit des pratiques concrètes appuyées de nombreux exemples tirés de cas d'entreprises à succès et enrichis de citations percutantes. Comme professeur d'université, j'en ai tiré satisfaction: un ouvrage bien structuré, documenté et qui s'inscrit dans des débats actuels en relations industrielles, plus particulièrement en gestion des ressources humaines. Et... si j'étais un praticien en gestion des ressources humaines, j'offrirais certes une copie de ce livre aux dirigeants de mon organisation ainsi qu'aux officiers du syndicat.

L'ouvrage se divise en trois parties. Dans la première partie, l'auteur expose sa thèse, propose des pratiques à mettre en œuvre, illustre leur efficacité et démontre la lenteur et le faible degré de leur diffusion, presque exclusivement aux
États-Unis. La deuxième partie traite des barrières et des difficultés reliées à l'implantation de ces pratiques visant le développement d'un avantage concurrentiel par l'entremise de la main-d'œuvre. La dernière partie du livre fait état de perspectives favorables aux changements, dont la promesse du mouvement qualité et quelques principes pouvant guider les changements organisationnels requis de manière à générer des transformations durables.

La première partie du livre, dès le début, met en évidence cinq compagnies (Southwest Airlines, Wal-Mart, Tyson Foods, Circuit City et Plenum Publishing). Ces entreprises ont deux éléments en commun. D'abord, bien qu'elles aient œuvré dans des environnements concurrentiels sectoriels hostiles pendant vingt ans, elles constituent le groupe des cinq firmes américaines affichant les actions les plus performantes (associées à des retours sur investissement élevés) pour la période de 1972 à 1992. Deuxièmement, selon l'auteur, la durabilité de leur succès à long terme n'est pas attribuable aux sources conventionnelles d'avantage concurrentiel (économies d'échelle, technologie, accès au financement, etc.), qui ont évolué dans le temps, mais bien à la façon dont elles ont géré leur maind'œuvre. D'où la thèse avancée par Pfeffer : obtenir un avantage concurrentiel par 
l'entremise de la main-d'œuvre constitue un facteur de différenciation crucial, difficile à imiter et durable, qui devient de plus en plus important. Cela oblige à modifier notre façon de penser: impliquer les gens plutôt que chercher à limiter l'étendue de leurs activités, et voir la main-d'œuvre comme un avantage stratégique et non pas comme un coût à minimiser ou à éviter. C'est dans cette perspective que l'auteur énonce une liste de seize "éléments critiques " (ou pratiques) pour une gestion efficace de la main-d'œuvre: la sécurité d'emploi, un recrutement sélectif, de hauts salaires, des primes de rendement, des employés propriétaires, le partage de l'information, la participation et l'empowerment, le travail en équipe et la réorganisation du travail, la formation, la polyvalence, l'égalitarisme symbolique, la compression des salaires, la promotion interne, une perspective à long terme, la mesure de performance de ces pratiques et une philosophie de gestion englobante. Pour élaborer ces pratiques et démontrer leur efficacité, il se sert d'arguments tirés de la littérature populaire et scientifique, d'entrevues avec plusieurs représentants de firmes d'une variété d'industries et enfin de l'application du sens commun.

Bien que l'auteur reconnaisse qu'il existe d'autres conditions de succès (stratégie de produit, technologie, expansion contrôlée, stratégie d'acquisition, structure de l'investissement), il ne met l'accent que sur " la gestion de la main-d'œuvre", sans insister sur les nuances à apporter selon les considérants stratégiques (menaces/opportunités, forces/faiblesses) pouvant guider leur implantation et affecter leur efficacité. Les effets des interrelations des pratiques et leur importance relative selon les contextes organisationnels et concurrentiels sont peu développés et laissés au jugement du lecteur. La majorité des preuves empiriques provient de témoignages, d'un nombre limité d'études de cas en profondeur et d'un faible nombre d'études quantitatives. Les principaux cas relatés se limitent au secteur privé, soit aux grandes entreprises des industries de l'automobile, du transport et du commerce de détail. Parmi les cinq entreprises mises en évidence au début du volume, seulement deux d'entre elles (Wal-Mart et Southwest Airlines) sont scrutées, sans toutefois adopter une approche longitudinale examinant en profondeur l'évolution de leurs sources d'avantages concurrentiels. Dans l'ensemble, l'approche de l'auteur tient plus de l'illustration que de la démonstration scientifique. Cette partie se termine par la démonstration que bien que ces pratiques ne soient pas particulièrement nouvelles, leur diffusion est lente et leur durabilité problématique dans les firmes américaines.

Dans la deuxième partie, l'auteur traite de plusieurs raisons de natures idéologique, institutionnelle et légale qui expliquent les difficultés reliées à l'implantation de ces pratiques. Plusieurs facteurs dissuadent ou empêchent les managers de mettre en place un système de gestion favorisant la coopération et la participation de la main-d'œuvre: parce qu'ils souscrivent à des théories du comportement humain qui présument la méfiance, le conflit et la supercherie (entre autres, celles portées par certains modèles économiques); parce qu'ils ont de "mauvais héros" (ceux qui obtiennent des résultats à court terme en luttant contre les syndicats et en effectuant des coupures draconniennes dans le capital humain); parce qu'ils utilisent un langage agressant (par exemple, downsizing, coupures, subordonnés). Une des grandes barrières à l'apprentissage des managers est certes l'héritage du passé qui entretient une vue étroite du contrôle dans les lieux de travail (l'influence du taylorisme) et des relations du travail (le modèle d'affrontement). Le cadre législatif du travail aux États-Unis fait aussi obstacle à l'introduction de changements permettant de transformer les ressources humaines en un avantage concurrentiel. Comparativement à d'autres pays industrialisés, les lois du travail ne favorisent 
pas la stabilité dans l'emploi, la formation, la participation et la syndicalisation des employés. Malheureusement, les firmes déploient peu d'effort pour réformer le cadre légal de manière à fournir un contexte social dans lequel des relations de coopération et de confiance entre les organisations et leurs employés seraient supportées par l'État. Plusieurs expériences rapportées dans le texte indiquent que le syndicat peut contribuer à l'innovation, à la productivité, ainsi qu'à instaurer une vision à long terme en contrepoids aux intérêts à court terme des banquiers et des actionnaires. Pour terminer cette deuxième partie de son volume, l'auteur met l'accent sur l'une des barrières importantes envers les changements proposés: la résistance venant de l'intérieur, celle des managers (craintes reliées à l'aplatissement de la hiérarchie, à la perte de statut et de privilèges, à la perte de pouvoir, à l'incertitude face à la transformation des rôles et face aux bénéfices du changement).

Ce qui constitue l'originalité de cette partie du volume traitant des barrières au changement, c'est le ton provocateur ainsi que la clarté et l'honnêteté du message adressé aux managers : le comportement du syndicat est conséquent à celui du management ; point n'est besoin d'attendre une réforme globale, car toute organisation progressiste peut, si elle le désire, offrir unilatéralement des garanties et des procédures qui visent à impliquer ses travailleurs et elle peut mettre en place des mécanismes internes pour faire face aux problèmes de relations d'emploi. De plus l'auteur précise que les résultats ne sont pas garantis et sont, du moins, fonction des actions du management. Cependant, la plupart des mesures à prendre qui sont mises de l'avant par Pfeffer pour triompher des barrières au changement reposent sur un agencement structuré d'anecdotes, de statistiques et de rappels théoriques guidé par le sens commun plutôt que sur des études scientifiques. Il faut toutefois reconnaître que le choix pertinent et la variété des illustrations éclairent le phénomène étudié.

Dans la troisième et dernière partie de son volume, Pfeffer fait l'éloge du " mouvement qualité ». Il souligne que les entreprises qui ont gagné le Baldrige Award (et d'autres qui ont adhéré à une démarche de qualité totale) sont celles qui font la promotion de pratiques et de comportements managériaux permettant d'obtenir un avantage concurrentiel par l'entremise des ressources humaines. Toutefois, ce prix de qualité ne contient pas d'indicateurs de rentabilité. De plus, l'auteur ne fait pas état des performances économiques des entreprises modèles retenues ni n'expose la durabilité de leur succès et du maintien de leurs pratiques dans le temps. Le mouvement qualité produit aussi deux autres effets positifs en faveur des changements au lieu de travail : il encourage les entreprises à apprendre les unes des autres, à examiner les approches propres à d'autres pays industrialisés et incite le gouvernement fédéral à soutenir l'implication et la formation des employés. Dans le dernier chapitre, l'auteur présente un diagnostic de cohérences interne et externe relatives aux politiques et aux pratiques de gestion des ressources humaines. Il propose ensuite des principes d'implantation, met en évidence des problèmes et des pièges à éviter. Puis, il expose les principaux rôles que devrait jouer la fonction gestion des ressources humaines dans la réalisation de l'avantage concurrentiel. Pfeffer termine son livre sur cette note : bien que les difficultés à surmonter soient nombreuses et la complexité des changements à effectuer indéniable, la compétition peut ne pas permettre d'autres choix.

Paradoxalement, deux éléments de la richesse de cet ouvrage peuvent rendre le lecteur suspicieux: une perspective centrée sur la gestion des ressources humaines et une approche dynamique dans la construction du volume. Premièrement, l'excellente couverture d'une gestion efficace des ressources humaines 
ne met en évidence que l'une des nombreuses dimensions importantes de la performance organisationnelle. Par exemple, Berggren ("Lean Production: The End of History ?", Work, Employment and Society, vol. 7, $\mathrm{n}^{\circ} 2,1993,163-188$ ) suggère que le régime de production a causé une résistance croissante des syndicats locaux et du désillusionnement chez les travailleurs de NUMMI (un cas important dans l'argumentation de Pfeffer) et ceux de CAMI alors que ces deux entreprises avaient adopté des pratiques de gestion des ressources humaines innovatrices. Une autre étude (R. Rehder, "Saturn, Uddevalla and the Japanese Lean Systems: Paradoxical Prototypes for the Twenty-first Century ", The Journal of Human Resource Management, vol. 5, $\mathrm{n}^{\circ} 1,1994,1-31$ ) soulève que Saturn et Uddevalla, deux entreprises avant-gardistes en matière de gestion des ressources humaines, ont fait face à des difficultés financières (Volvo a annoncé la fermeture de l'usine Uddevalla en 1993). Deuxièmement, une étude en profondeur sur l'évolution dans le temps et sur les effets des diverses stratégies et tactiques concurrentielles des cinq entreprises les plus performantes de 1972 à 1992 citées au début (ou des entreprises primées au Baldrige Award) permettrait probablement de mettre en perspective la thèse avancée par Pfeffer. Un autre élément méthodologique à envisager, quoique négligé dans les illustrations de l'auteur, pourrait consister à recueillir avec une plus grande attention le point de vue des travailleurs en ce qui a trait à la façon dont ils sont "gérés". Ceci peut fournir de l'information utile pour évaluer l'impact des pratiques mises en ceuvre sur les relations avec les employés, leur satisfaction, leur motivation et surtout de "relâcher le pouvoir des ressources humaines".

Somme toute, Jeffrey Pfeffer atteint son objectif. En plus de bien informer le public à qui il s'adresse de manière provocante et convaincante, de susciter des débats dans le monde universitaire, il redonne espoir aux praticiens en gestion des ressources humaines car, désormais, les managers peuvent difficilement nier qu'ils ont la responsabilité et la possibilité d'entreprendre la route qui mène au développement d'un avantage stratégique difficile à imiter et durable: le pouvoir et la force des ressources humaines. Pour effectuer un premier pas dans cette direction, Pfeffer propose que le management adopte un langage approprié. Ainsi, l'expression héritée du passé "gérer les ressources humaines " pourrait peut-être désormais s'énoncer, telle qu'utilisée une fois dans le volume, "gérer avec les gens".

\section{FRANÇOIS VILLENEUVE}

Université du Québec en Abitibi-Témiscamingue

\section{Les secrets de la préparation financière à la retraite}

par Louis ASCAH, Sherbrooke : Éditions du CRP, 1996, 189 p., ISBN 2-92085997-8.

Ce livre a pour objectif de rendre accessible la préparation de la retraite aux personnes intéressées. L'auteur atteint son objectif. Tout en étant rigoureux, il permet au lecteur de se faire une bonne idée sur les différents aspects nécessaires à une bonne planification de la retraite, c'est-à-dire les besoins à la retraite, les modes d'épargne public et privé, ainsi que les placements pour faire fructifier cette épargne. Par un heureux mélange de textes et de notes techniques, il trace un juste portrait de la planification à la retraite. Ce livre sera utile au lecteur qui désire être bien informé pour prendre des décisions éclairées.

Il fournit des outils utiles pour permettre au lecteur de mieux comprendre des données qui sont transmises par les conseils en placement, les institutions financières ou les spécialistes en 